\title{
The Neural Code of Auditory Phantom Perception
}

\author{
Nathan Weisz, ${ }^{1,2}$ Simona Müller, ${ }^{1}$ Winfried Schlee, ${ }^{1}$ Katalin Dohrmann, ${ }^{1}$ Thomas Hartmann, ${ }^{1}$ Thomas Elbert ${ }^{1}$ \\ ${ }^{1}$ Department of Psychology, University of Konstanz, D-78464 Konstanz, Germany, and ${ }^{2}$ Institut National de la Santé et de la Recherche Médicale, Unité \\ 280, 69500 Lyon, France
}

Tinnitus is defined by an auditory perception in the absence of an external source of sound. This condition provides the distinctive possibility of extracting neural coding of perceptual representation. Previously, we had established that tinnitus is characterized by enhanced magnetic slow-wave activity $(\sim 4 \mathrm{~Hz})$ in perisylvian or putatively auditory regions. Because of works linking high-frequency oscillations to conscious sensory perception and positive symptoms in a variety of disorders, we examined gamma band activity during brief periods of marked enhancement of slow-wave activity. These periods were extracted from 5 min of resting spontaneous magnetoencephalography activity in 26 tinnitus and 21 control subjects. Results revealed the following, particularly within a frequency range of $50-60 \mathrm{~Hz}$ : (1) Both groups showed significant increases in gamma band activity after onset of slow waves. (2) Gamma is more prominent in tinnitus subjects than in controls. (3) Activity at $\sim 55 \mathrm{~Hz}$ determines the laterality of the tinnitus perception.

Based on present and previous results, we have concluded that cochlear damage, or similar types of deafferentation from peripheral input, triggers reorganization in the central auditory system. This produces permanent alterations in the ongoing oscillatory dynamics at the higher layers of the auditory hierarchical stream. The change results in enhanced slow-wave activity reflecting altered corticothalamic and corticolimbic interplay. Such enhancement facilitates and sustains gamma activity as a neural code of phantom perception, in this case auditory.

Key words: tinnitus; phantom perception; gamma band activity; slow-wave activity; magnetoencephalography; spontaneous activity

\section{Introduction}

Approximately 5-15\% (Eggermont and Roberts, 2004) of the population have auditory percepts, termed tinnitus, in the absence of physical input. Ongoing neural activity that is common in tinnitus, but sparse in controls, should therefore be a likely candidate of the neural coding of sensory representations. Therefore, tinnitus provides a unique model to advance understanding of the neural code of sensory representations, perceptions and positive symptoms.

Usually tinnitus is triggered by damage to the peripheral hearing system, leading to a readjustment of central auditory activity (Irvine et al., 2001). Because of the richness of feedforward and feedback connections, changes spread throughout the processing hierarchy. Following tinnitus-inducing interventions, reorganization could be demonstrated in animals from as low as the dorsal cochlear nucleus up to the auditory cortex (Eggermont and Roberts, 2004). It is assumed that deafferented neuronal cell assemblies lose their ability to inhibit normally silent excitatory input from neighboring regions on tonotopic maps. Map reorganization (Calford et al., 1993; Rajan and Irvine, 1998), defined by a representational expansion of these neighboring regions, would be an indication of this process. However, map reorganization

Received Aug. 25, 2006; revised Dec. 6, 2006; accepted Dec. 7, 2006.

This work was supported by Deutsche Forschungsgemeinschaft Grant EL 101/20. Helpful input concerning signal analysis was given by Andreas Keil and Christian Wienbruch. Christina Roberts kindly proofread this manuscript.

Correspondence should be addressed to Nathan Weisz at his present address, Institut National de la Santé et de la Recherche Médicale, Unité 280, Centre Hospitalier Le Vinatier, Bâtiment 45295 Boulevard Pinel, 69500 Bron, France. E-mail: weisz@lyon.inserm.fr.

DOI:10.1523/JNEUROSCI.3711-06.2007

Copyright $\odot 2007$ Society for Neuroscience $\quad$ 0270-6474/07/271479-06\$15.00/0 only becomes apparent during neural activation, whereas tinnitus may be a permanent perception.

A magnetoencephalography (MEG) study by our group demonstrated altered spontaneous activity in a group of tinnitus sufferers compared with controls (Weisz et al., 2005), characterized by a reduction of the $\alpha(\sim 10 \mathrm{~Hz})$ rhythm and a concomitant increase of slow-wave $(<4 \mathrm{~Hz} ; \delta)$ activity, particularly in perisylvian regions. Slow waves are typical of cortical regions disconnected from thalamocortical afferents (Steriade, 2006). Furthermore, slow waves on a cortical level can be enhanced by low-threshold spike bursts, generated in thalamic nuclei when hyperpolarized during deafferentation or overinhibition. These bursts that occur in a regular rhythm of $\sim 4 \mathrm{~Hz}$ (Jeanmonod et al., 1996) have been coined clinically as thalamocortical dysrythmia (TCD) (Llinas et al., 1999). These disorders are marked by the presence of positive symptoms, i.e., excessive amount of thoughts, behaviors, or sensory phantom perceptions.

Yet $\delta$ activity does not have to be accompanied by positive symptoms. It is also found in stroke (Meinzer et al., 2004) or Alzheimer patients (Fernandez et al., 2002). Based on previous works linking conscious visual perception to gamma oscillations (Llinas et al., 1998; Singer, 1999), it is plausible to assume that positive symptoms may arise through the concerted action of slow- and high-frequency oscillations. Enhanced gamma activity may occur in circumscribed cortical zones between deafferented and normally afferented regions, resulting from a loss of lateral inhibition of the former. This hypothesis is termed "edge effect" within the TCD framework. Empirical evidence comes from works using voltage-sensitive dyes (Llinas et al., 2005) and bicoherence in patients undergoing neurosurgery (Sarnthein et al., 
Table 1. Information on tinnitus participants

\begin{tabular}{|c|c|c|c|c|c|c|c|c|}
\hline Subject & Sex & Age & T distress & Etiology & T duration & T side & HL/TEN left & $\mathrm{HL} / \mathrm{TEN}$ right \\
\hline 1 & M & 29 & NA & Unknown & 1 & $\mathrm{RD}$ & 5 & 4 \\
\hline 2 & $\mathrm{~F}$ & 38 & 20 & Sudden hearing loss & 14 & $\mathrm{RD}$ & 4 & 13 \\
\hline 3 & $\mathrm{~F}$ & 32 & 5 & Unknown & 2 & $\mathrm{R}$ & 0 & 4 \\
\hline 4 & M & 20 & 32 & Unknown & 2 & $\mathrm{RD}$ & 10 & 7 \\
\hline 5 & M & 24 & 22 & Noise trauma & 3 & $\mathrm{R}$ & 15 & 0 \\
\hline 6 & $\mathrm{~F}$ & 22 & 8 & Unknown & 6 & B & 8 & 0 \\
\hline 7 & $M$ & 23 & 3 & Noise trauma & 3 & B & 7 & 8 \\
\hline 8 & M & 26 & 21 & Lyme disease & 9 & B & 6 & 0 \\
\hline 9 & $\mathrm{~F}$ & 25 & 4 & Unknown & 6 & LD & 2 & 8 \\
\hline 10 & $\mathrm{~F}$ & 63 & 51 & VIII nerve transection after tumor & 17 & B & 50 & deaf \\
\hline 11 & M & 58 & 14 & Occupational noise & 14 & $\mathrm{~L}$ & $<25$ & 65 \\
\hline 12 & M & 52 & 21 & Sudden hearing loss & 8 & $\mathrm{~L}$ & 50 & $<25$ \\
\hline 13 & M & 57 & 30 & Occupational noise & 12 & $\mathrm{RD}$ & 55 & 55 \\
\hline 14 & M & 60 & 13 & Occupational noise & 4 & LD & 53 & 55 \\
\hline 15 & M & 65 & 21 & Unknown & 3 & $\mathrm{~L}$ & 38 & 70 \\
\hline 16 & M & 60 & 68 & Noise trauma & 10 & B & 68 & 52 \\
\hline 17 & M & 51 & 60 & Sudden hearing loss; stress & 1.5 & $\mathrm{~L}$ & 44 & 52 \\
\hline 18 & M & 55 & 45 & Mandibular disorder & 15 & B & 35 & 40 \\
\hline 19 & M & 34 & 18 & Sudden hearing loss after dental treatment & 4 & $\mathrm{~L}$ & 49 & 46 \\
\hline 20 & M & 33 & 19 & Hereditary & 5 & B & NA & NA \\
\hline 21 & M & 52 & 20 & Sudden hearing loss & 1.5 & B & 36 & 36 \\
\hline 22 & M & 53 & 50 & Stress & 1 & B & NA & NA \\
\hline 23 & M & 58 & 22 & Stress & 10.5 & $\mathrm{R}$ & 55 & 50 \\
\hline 24 & M & 61 & 35 & Unknown & 7 & B & 70 & 69 \\
\hline 25 & $\mathrm{~F}$ & 58 & 38 & Stress & 15 & L & NA & NA \\
\hline 26 & M & 63 & 47 & Stress & 1 & LD & NA & NA \\
\hline
\end{tabular}

Tinnitus distress (T distress) was assessed using the Tinnitus Questionnaire. Each score can be associated to a distress type (mild: 0 -30; moderate: 31-46; severe: 47-59; very severe: 60-84). The column Etiology indicates the tinnitus-eliciting event as described by the subject. For one subject, the distress score was not obtained (indicated by NA). Tinnitus duration ( $T$ duration) was measured in years. Laterality of the tinnitus percept ( $T$ side) could be either fully unilateral [left (L); right (R)], bilateral but with a dominant side [left dominant (LD); right dominant (RD)], or bilaterally equal (B). Regarding hearing thresholds, subjects 1-9 were tested using the TEN method (here maximum threshold given in decibels above noise level). For all other subjects for which data are available, standard clinical audiograms were assessed (NA indicates missing data). M, Male; $F$, female; $H L$, hearing loss.

2006). Although the former is an in vitro demonstration, the latter lacks time information, making statements about the dynamic interplay between low and high frequencies difficult. Using wavelet and power spectrum analysis of ongoing neuromagnetic activity triggered to the onset of slow waves, we attempted to overcome these shortcomings.

\section{Materials and Methods}

Subjects. Twenty-six tinnitus participants took part in the study (20 male, 6 female; average age, 45 years; range, $20-65$ years). They were recruited via newspaper ads and flyers posted at the University of Konstanz. The data were compared with those of 21 control subjects reporting no tinnitus or other hearing disorder ( 16 males, 5 females; average age, 35 years; range, 23-78 years). Regarding laterality of tinnitus loudness (or dominance in case of bilateral tinnitus), 9 subjects reported left, 10 bilaterally equal, and 7 right lateralized tinnitus. Average tinnitus duration was 6.75 years $( \pm 1.01)$. Tinnitus distress was assessed using the German version of the Tinnitus Questionnaire (Goebel and Hiller, 1998). The average distress score was $27.48( \pm 3.54)$, which corresponds to mild distress. In the majority of cases, tinnitus subjects reported etiologies related to hearing damage as elicitor of their tinnitus. This is partly reflected in those subjects for which hearing thresholds were available, which were measured either using a standard clinical audiogram or the Threshold Equalizing Noise (TEN) test (Moore et al., 2000). Because the procedure for the TEN test resembles that reported recently, we do not give a detailed account of this test (Weisz et al., 2006). All hearing threshold data were obtained on an Interacoustics AC40 clinical audiometer (Audiometrics, Shreveport, LA). Tested frequencies were $0.25,0.5,1,1.5,2,3,4,5,6$, and $8 \mathrm{kHz}$. Table 1 summarizes information regarding our tinnitus group. Participants were acquainted with the procedure and aims of the study, and written informed consent was obtained. The study was approved by the Ethical Committee of the University of Konstanz.

Procedure and data acquisition. Five minutes of resting MEG were recorded using a 148 channel whole-head magnetometer (Magnes 2500
WH; 4-D Neuroimaging, San Diego, CA) in a magnetically shielded chamber (Vakuumschmelze, Hanau, Germany). The data stream was sampled with $678.17 \mathrm{~Hz}$ and filtered online from 1-200 Hz. Participants lay comfortably in a supine position and were told to focus on a fixed point $\sim 1.5 \mathrm{~m}$ away. They were instructed to keep their eyes open and to remain relaxed. The investigators were able to monitor the participants on a video screen to assure that they kept their eyes open. Eye movements [electro-oculogram (EOG)] were recorded with four electrodes: two were placed at the left and right outer canthi (horizontal EOG) and two above and below the right eye (vertical EOG). Electrocardiogram was recorded from two electrodes placed on the left and right forearm. To determine the head position within the MEG helmet, positions of five index points and individual head shapes were sampled using a digitizer.

Data analysis. Continuous data were corrected for eye and heartbeatrelated artifacts using a multiple source approach (Berg and Scherg, 1994) implemented in BESA (MEGIS Software, Gräfelfing, Germany). The data were then projected from the 148 sensors onto a montage consisting of eight sources. In this approach, a source model is generated. Apart from two sources placed into the regions of interest, i.e., left and right auditory cortex, six sources were distributed over the cortex: left/ right prefrontal cortex, anterior cingulum, left/right parietal cortex, and occipital cortex. It is important to emphasize that locations of the sources were predefined by the authors and should not be confused with statements regarding fine-grained spatial localization of activity that can be drawn from functional neuroimaging studies. The locations outside of the regions of interest were chosen such that they coarsely covered the entire volume and were identical for all subjects. The presence of sources outside the region of interest reduced the contributions of these regions to auditory sources and acted as a kind of spatial filter. The strength of using source montages is to obtain time courses of brain activities from distinct (coarsely defined) brain regions (Scherg et al., 2002; Bledowski et al., 2006). After this step, data were downsampled to $450 \mathrm{~Hz}$ and exported for additional analysis in Matlab (The MathWorks, Natick, MA). Remaining movement and muscle artifacts were removed from the contin- 


\section{Left Temporal}
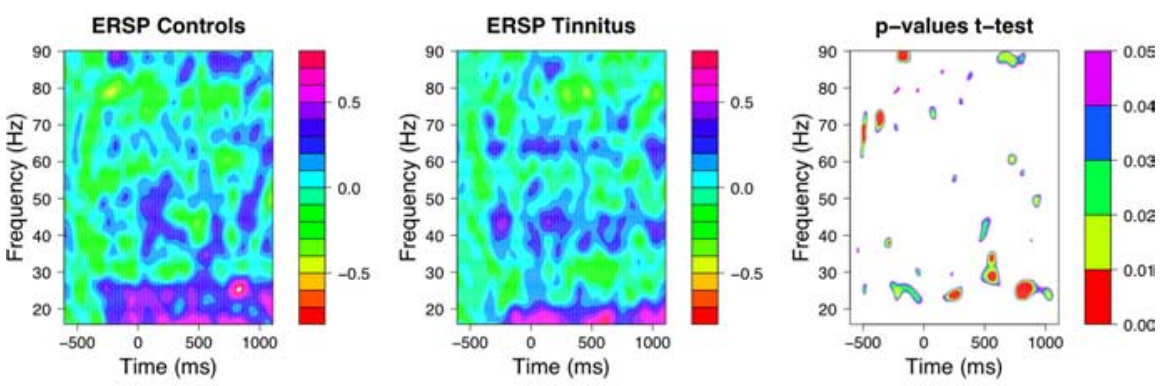

Right Temporal
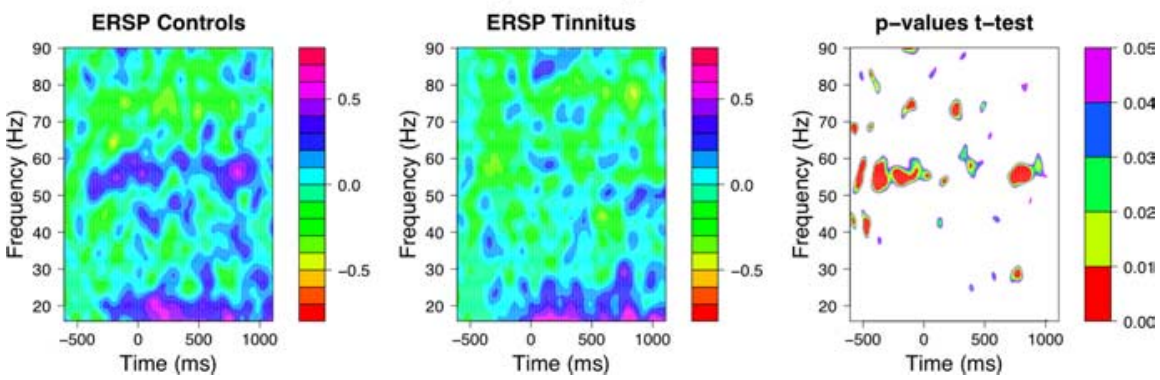

Figure 1. Time-frequency representation of high-frequency oscillatory activity triggered to the onset of SWA. Top panels depict ERSPs for both groups and $p$ values derived from $t$ tests for the left temporal source, whereas the bottom panels show this for the right temporal source. Note that a difference between the groups can be seen at $50-60 \mathrm{~Hz}$, particularly for the right temporal source. The reason for this difference can be seen more clearly in Figure 2 .

uous data by visual inspection using visualization tools supplied by EEGLAB (Delorme and Makeig, 2004).

As a first step, we analyzed the data analogously to our previous work (for details, see Weisz et al., 2005), i.e., over the entire 5 min length. Normalized power (see also below) was compared at auditory sources between the groups for the following bands: $\delta(1-3 \mathrm{~Hz}), \alpha(8-12 \mathrm{~Hz})$, and gamma (40-90 Hz; excluding 49-51 Hz). Factors of the ANOVA were group and hemisphere. Gamma activity was not reported in the previous study, because a strong downsampling did not permit analysis in this frequency band.

To obtain time points with increases in slow-wave activity (SWA), the remaining data were bandpass filtered at $2-7 \mathrm{~Hz}$ and Hilbert transformed. The squared modulus of the time series of complex numbers was calculated to yield the continuous power. Subsequently, time points with peaks were identified. In a first step, time points with power values lower than the median peak power were discarded from additional analysis. In the next step, we also discarded all time points in which an additional peak larger than the median occurred in a time window of $750 \mathrm{~ms}$ before. This was done to ensure that chosen time points represent a genuine increase in SWA relative to a preceding time period. On average, this approach yielded $\sim 30$ peaks per participant, fulfilling the specified criteria for the left and right temporal source. These time points were used to cut out time windows from the non-Hilbert-transformed data stream of $750 \mathrm{~ms}$ pre-SWA peak and $1250 \mathrm{~ms}$ post-SWA peak. This time window is long enough to incorporate a few cycles even at the lowest frequency ( 2 $\mathrm{Hz}$ corresponding to 4 cycles during the $2 \mathrm{~s}$ ).

These time windows (epochs) were subsequently submitted to a wavelet analysis using the timef function implemented in EEGLAB. Generally, wavelet analysis yields a time-frequency representation of the data, allowing for the investigation of spectral changes at distinct time points [event-related spectral perturbations (ERSPs)] (Delorme and Makeig, 2004). Here, changes in power are expressed relative to a baseline (i.e., output in decibels), which was defined as $750-375$ ms pre-SWA. This baseline ensures that, even at low frequencies, no post-SWA activity is wrongly incorporated into the baseline because of the temporal resolution properties (lower at low frequencies) of the wavelets. Additionally, we cross-correlated the ERSPs of all frequencies with that of the SWA to confirm that the time course of certain high frequencies was significantly associated to SWA. To determine time-frequency clusters with significant ERSP/cross-correlation difference between groups, each time-frequency point was compared via an unpaired $t$ test.

Because ERSPs yield values relative to baseline, it is not possible to compare whether differences between groups already exist during this time period. For this purpose, we estimated the spectral power during baseline and a $0-500$ ms post-SWA time window using fast Fourier transformation (FFT). To normalize for the strong interindividual variability regarding absolute power, we divided the power for each frequency by the mean value of the average preSWA and post-SWA power spectrum of each individual. This gives the relative content of a certain frequency to the overall power spectrum but leaves pre-SWA and post-SWA differences unchanged. The results of this analysis were related to tinnitus laterality. This was performed via ANOVAs with a linear trend, assuming that dominance in the gamma band should be expressed contralateral to the tinnitus percept in unilateral cases and will be expressed equally across hemispheres in tinnitus perceived equally strong in both ears. Hemispheric dominance was operationalized using a laterality index: $(\mathrm{LH}-\mathrm{RH}) /(\mathrm{LH}+\mathrm{RH})$, where $\mathrm{RH}$ and $\mathrm{LH}$ normalized power values for the right and left hemisphere, respectively.

Although the groups differed significantly in age, no associations between age and power in the relevant frequency bands could be detected (all $p$ values $>0.29$ ). Therefore, we do not consider age to play an important role in our study.

\section{Results}

\section{Ongoing spontaneous activity}

The results for $\delta$ and $\alpha$ remain largely unchanged compared with Weisz et al. (2005). The ongoing spontaneous activity in tinnitus participants is marked by enhanced $\delta\left(F_{(1,90)}=4.52 ; p<0.04\right)$ and reduced $\alpha\left(F_{(1,90)}=17.08 ; p<.001\right)$ activity in auditory brain regions. Additionally to these two known effects, we found a significant increase of gamma activity in the tinnitus group $\left(F_{(1,90)}=10.54 ; p<.002\right)$. No effect of hemisphere was observed for any band.

\section{Wavelet and cross-correlation}

Epochs were extracted from the continuous data stream, with an increase in SWA $(2-7 \mathrm{~Hz})$ at $0 \mathrm{~s}$ and low SWA during a $750 \mathrm{~ms}$ pre-SWA period (see Materials and Methods for details). These epochs were submitted to time-frequency analysis using wavelets, yielding ERSPs. The results are displayed separately for the groups and the right and left source in the auditory regions (Fig. 1). In the left hemisphere, no striking differences can be observed between the groups, as seen by the two-dimensional representation of $p$ values on the right-hand side of Figure 1. This is different, however, for the right hemisphere, in which temporary wideranging differences can be detected between 50 and $60 \mathrm{~Hz}$. This is attributable to a different evolvement of power in the two groups (Fig. 2). Whereas control subjects exhibit an enhancement of gamma band activity (GBA) starting from $\sim 500 \mathrm{~ms}$ before SWA and reaching a peak approximately simultaneously to the SWA peak, tinnitus subjects show a transient decrement of GBA start- 
ing at about the same time and reaching a minimum at around 250 ms pre-SWA. Overall, 50-60 Hz GBA appears to be more similar in its time course to that of the SWA for controls compared with tinnitus participants. Thus, there is an apparent modulation of the GBA by the SWA in the controls but not in tinnitus subjects.

The differences indicated by Figures 1 and 2 are substantiated by cross-correlating the wavelet ERSP output for SWA with those for the higher frequencies (Fig. 3). A strong difference between the groups is particularly observable between 55 and $60 \mathrm{~Hz}$ in the right hemisphere. This stems from higher cross-correlations, with a maximum close to zero (lag) in the control group, indicating that time series between SWA and gamma $(55-60 \mathrm{~Hz})$ activity are more similar in these subjects.

\section{Power spectrum}

The results described above raises the question as to whether the absolute power of GBA around SWA differs between the groups. This is a result of the methodology used because ERSPs yield changes for each frequency relative to its baseline. Therefore, we also calculated power spectra during baseline (750-375 ms preSWA) and in a time window after SWA onset $(0-500 \mathrm{~ms}$ post-SWA).

To test the hypothesis that tinnitus subjects may already exhibit enhanced GBA during baseline, we extracted the averaged normalized power between $55-60 \mathrm{~Hz}$, i.e., the band in which controls showed a significant co-modulation with SWA (see Fig. 4). Statistical analysis revealed main effects for time $\left(F_{(1,45)}=\right.$ $237.22 ; p<.001)$ and group $\left(F_{(1,45)}=5.36 ; p<0.03\right)$. The time effect is attributable to a $\sim 33 \%$ increase of gamma from preSWA onset to post-SWA onset, whereas the group effect reflects a $\sim 30 \%$ enhanced gamma activity in the tinnitus group relative to control subjects.

\section{Effect of tinnitus laterality}

The results demonstrate enhanced GBA in tinnitus sufferers. Yet to be a potential correlate of the phantom perception, group differences are not sufficient; there should also be perceptual differences within the tinnitus group. Based on individual reports concerning tinnitus laterality, we compared the magnitude of the laterality index at all frequencies pre- $\delta$ and post- $\delta$. We assumed that frequencies relevant for perception should yield a significant effect in an ANOVA applying a linear contrast. This was the case for the $55 \mathrm{~Hz}$ region (Fig. 5) $(t=2.90 ; p<.008)$ while right dominant tinnitus is reflected in left lateralized $55 \mathrm{~Hz}$ activity. The opposite occurs for left dominant tinnitus. Subjects with bilateral equally strong tinnitus exhibit a laterality index close to zero, indicating absence of a hemispheric dominance.

\section{Discussion}

The main goal of our work was to characterize the high-frequency oscillatory dynamics in tinnitus that occurs within distinct episodes of slow-wave activity, which is overall enhanced in tinnitus (Weisz et al., 2005). We were inspired by various lines of research suggesting that enhanced control group.
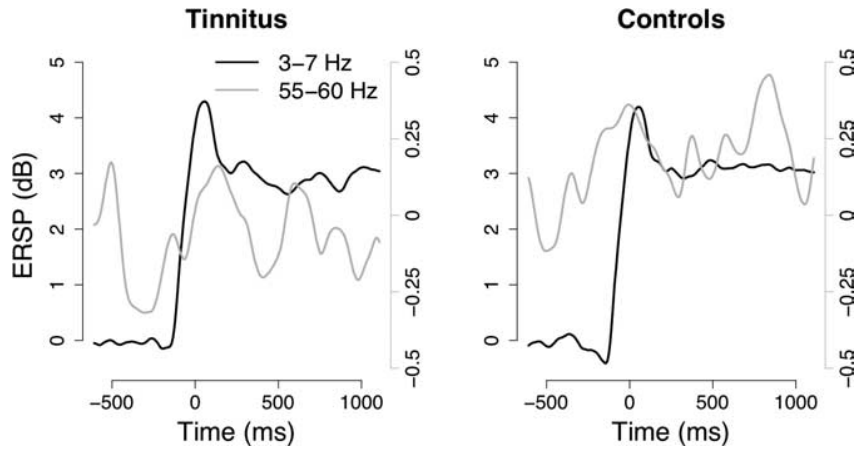

Figure 2. Overlay of SWA (black line; left $y$-axis) and 55-60 Hz activity (gray line; right $y$-axis) for tinnitus and control subjects at the right temporal source. Control subjects in particular show a clear increase of gamma activity along with SWA, starting before SWA onset. In the same window, tinnitus subjects reduce their gamma activity, leading to the significant group difference observed in Figure 1.

GBA (1) may be the cortical expression of binding features into conscious visual percepts (Singer, 1999) and (2) may clinically underlie phantom perceptions in general (Llinas et al., 1999). On a cellular level, GBA is related to synchronous spike discharges of neurons (Singer, 1999). Synchronicity is considered to be a mechanism enhancing the salience of this neuronal activity, favoring additional processing at subsequent stages of the cortical hierarchy involved in perception. GBA may reflect coherent activity of spatially separated neuronal assemblies binding various features of one object, such as a face, or coherent activity within one assembly coding the same feature. It seems likely that spatially restricted synchronous activity generates the oftentimes very simple auditory percepts that characterize tinnitus (e.g., a tone or narrow-band noise). This has been partially demonstrated in an animal study (Norena and Eggermont, 2003) that

\section{Left Temporal}
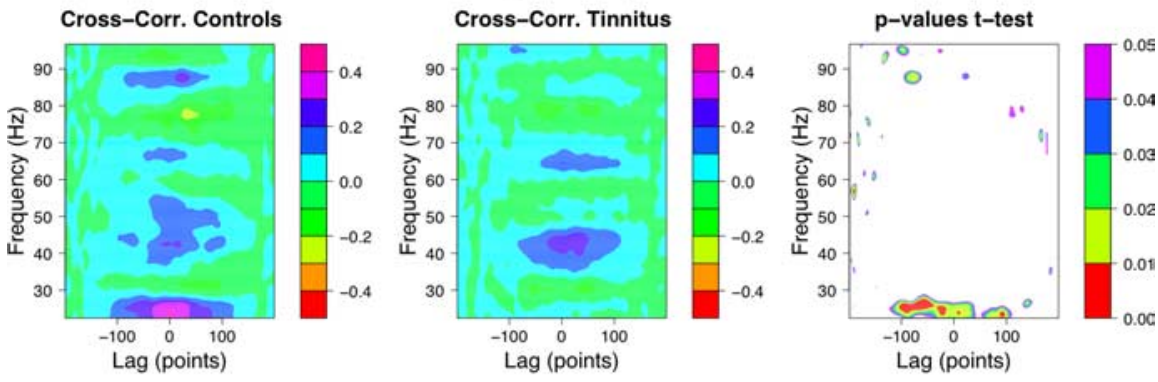

\section{Right Temporal}
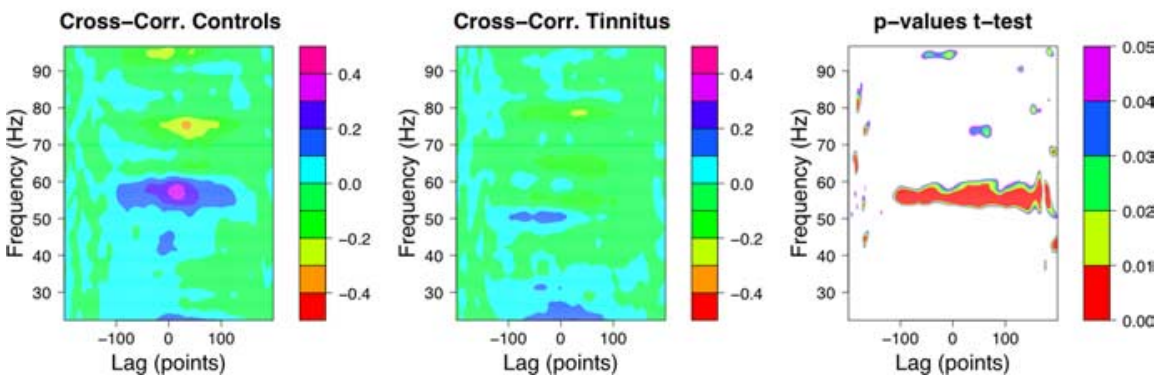

Figure 3. Time-frequency representation of cross-correlation (Cross-Corr.) between the SWA-ERSP and the ERSPs of the other frequencies. A marked difference can be seen between the groups, particularly at the right temporal source, reflecting higher cross-correlation between SWA and $\sim 55 \mathrm{~Hz}$ in the control group. This indicates that these time series are more similar in the 


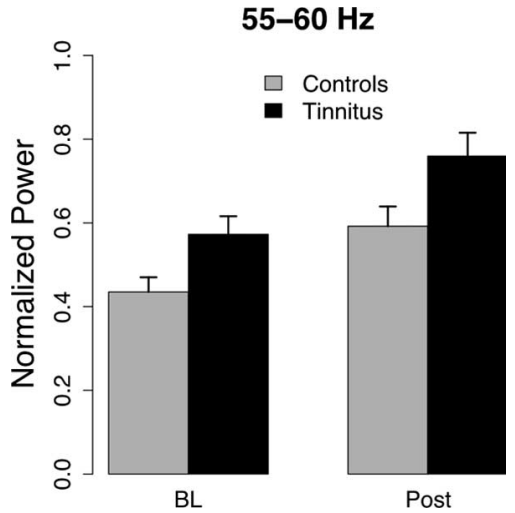

Figure 4. Normalized power spectrum averaged ( \pm SE) over left and right temporal sources shows that gamma activity increases after onset of strong SWA in both groups. Generally, however, the content of $55-60 \mathrm{~Hz}$ activity to the overall power is increased in tinnitus. $\mathrm{BL}$, Baseline.

shows synchronous spiking within deafferented regions immediately after noise trauma. The relationship to gamma band activity was, however, not shown.

Our results do demonstrate that the high-frequency activity is enhanced in the tinnitus group compared with the nontinnitus group and that $55 \mathrm{~Hz}$ activity is, at least in temporal proximity to strong SWA, a particular indicator of the dominance of tinnitus laterality. This is an important criterion indicating in fact that this oscillatory activity may be the fundamental perceptual correlate of phantom sound perception. Although the laterality effect involves a circumscribed frequency, it is nonetheless likely meaningful, because it is the exact frequency that proves to be slowwave modulated, particularly in healthy control subjects (Figs. $1-3)$. The latter result in awake control participants validates Llinas' (Llinas et al., 2005) basic idea that fast oscillations can be triggered by slow-wave activity. However, this transient release of inhibition does not lead to tinnitus in normal hearing subjects.

Interestingly, when we take wavelet and cross-correlation data into account, the time courses of SWA and GBA appear to be even more similar in controls than in tinnitus. In the latter group, gamma does not seem to be up-modulated to the same extent as in controls (the pre-post difference in the power spectrum could be the effect of transient reductions during baseline). Nevertheless, tinnitus subjects exhibit a chronically increased level of slowwave activity (Weisz et al., 2005), indicating a more stable release from inhibition, reflected in the overall enhanced level of GBA. After a long history of deafferentation, it is likely that the synaptic strength between neurons being part of the tinnitus network underlying percept increases. GBA becomes self-sustained to a certain degree, because an activation of a smaller amount of neurons is now necessary to ignite the representational network. Therefore, it is hypothesized that GBA is already normally close to upper limits in tinnitus and that more SWA will not enhance it to the same extent as in auditory cortex with regular input. This stable self-sustaining reorganized state could be the reason that tinnitus has proven to be very therapy-resistant thus far. Support for this comes from our finding that GBA $(40-90 \mathrm{~Hz})$ is also elevated when the entire data stream (i.e., non-SWA triggered) is taken into account. It will be interesting to scrutinize the role of auditory $\alpha$ activity in the future that could reflect an active inhibitory mechanism, which is unsustainable in regions marked by SWA.

We conclude that tinnitus is a condition triggered by a deafferentation or disconnection of cortical cell assemblies that possess representational functions, with the most common cause

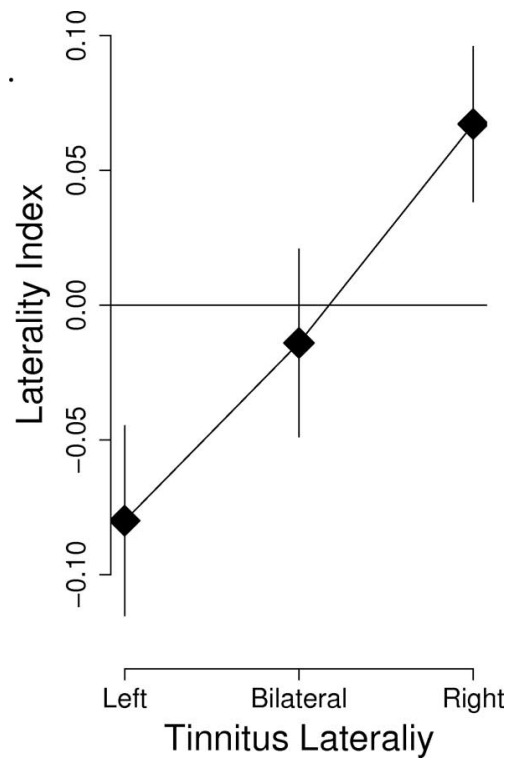

Figure 5. Tinnitus laterality is reflected by $55 \mathrm{~Hz}$ oscillatory activity as measured by the laterality index (mean \pm SE). Participants with right lateralized tinnitus have left dominant 55 $\mathrm{Hz}$ activity and vice versa in left lateralized tinnitus. Bilateral tinnitus subjects exhibit a laterality index close to zero, indicating absence of a hemispheric dominance of $55 \mathrm{~Hz}$ activity.

being cochlear damage (Norena et al., 2002; König et al., 2006; Weisz et al., 2006). As can be taken from Table 1, 72\% (16 of 22) of the tinnitus subjects exhibit an audiometrically derived hearing loss (if a conventional $25 \mathrm{~dB}$ hearing level criteria is taken for the clinical audiogram; for the TEN test, a value $\geq 10 \mathrm{~dB}$ is likely to reflect a cochlear zone in which inner-hair cells are no longer functioning). Three subjects assessed via TEN test exhibited borderline values ( $8 \mathrm{~dB}$; normal would be a threshold close to masking level, i.e., close to $0 \mathrm{~dB}$ ), indicating that some damage is present although respective regions of the cochlea cannot be described as completely "dead." Therefore, it appears unlikely that tinnitus can be modeled without taking hearing damage into account (see also Weisz et al., 2006). König et al. (2006) showed that even two hearing loss groups with and without tinnitus show distinct audiometric differences. Particularly, the slope of hearing loss appears to be sharper in tinnitus, which could mean that the relevant neuronal reorganizational processes eventually take place at points with steep activational discontinuities. In our framework, deafferentation then leads to a corticothalamic and putatively corticolimbic interplay resulting in enhanced slow oscillatory activity of these cell assemblies. Slow-wave activity sustains high-frequency oscillations (perhaps indirectly by release of inhibition, normally sustained by the $\alpha$ rhythm) reflecting spatially restricted synchronized firing of neurons underlying the tinnitus percept. On a general level, our work demonstrates how oscillatory activity may be intrinsically related to conscious perception in the absence of external stimulation.

\section{References}

Berg P, Scherg M (1994) A multiple source approach to the correction of eye artifacts. Electroencephalogr Clin Neurophysiol 90:229-241.

Bledowski C, Cohen Kadosh K, Wibral M, Rahm B, Bittner RA, Hoechstetter K, Scherg M, Maurer K, Goebel R, Linden DE (2006) Mental chronometry of working memory retrieval: a combined functional magnetic resonance imaging and event-related potentials approach. J Neurosci 26:821-829.

Calford MB, Rajan R, Irvine DR (1993) Rapid changes in the frequency tuning of neurons in cat auditory cortex resulting from pure-toneinduced temporary threshold shift. Neuroscience 55:953-964. 
Delorme A, Makeig S (2004) EEGLAB: an open source toolbox for analysis of single-trial EEG dynamics including independent component analysis. J Neurosci Methods 134:9-21.

Eggermont JJ, Roberts LE (2004) The neuroscience of tinnitus. Trends Neurosci 27:676-682.

Fernandez A, Maestu F, Amo C, Gil P, Fehr T, Wienbruch C, Rockstroh B, Elbert T, Ortiz T (2002) Focal temporoparietal slow activity in Alzheimer's disease revealed by magnetoencephalography. Biol Psychiatry 52:764-770.

Goebel G, Hiller W (1998) Tinnitus-Fragebogen (TF): Ein Instrument zur Erfassung von Belastung und Schweregrad bei Tinnitus. Göttingen: Hogrefe.

Irvine DR, Rajan R, Brown M (2001) Injury- and use-related plasticity in adult auditory cortex. Audiol Neurootol 6:192-195.

Jeanmonod D, Magnin M, Morel A (1996) Low-threshold calcium spike bursts in the human thalamus. Common physiopathology for sensory, motor and limbic positive symptoms. Brain 119:363-375.

König O, Schaette R, Kempter R, Gross M (2006) Course of hearing loss and occurrence of tinnitus. Hear Res 221:59-64.

Llinas R, Ribary U, Contreras D, Pedroarena C (1998) The neuronal basis for consciousness. Philos Trans R Soc Lond B Biol Sci 353:1841-1849.

Llinas R, Urbano FJ, Leznik E, Ramirez RR, van Marle HJ (2005) Rhythmic and dysrhythmic thalamocortical dynamics: GABA systems and the edge effect. Trends Neurosci 28:325-333.

Llinas RR, Ribary U, Jeanmonod D, Kronberg E, Mitra PP (1999) Thalamocortical dysrhythmia: a neurological and neuropsychiatric syndrome characterized by magnetoencephalography. Proc Natl Acad Sci USA 96:15222-15227.

Meinzer M, Elbert T, Wienbruch C, Djundja D, Barthel G, Rockstroh B
(2004) Intensive language training enhances brain plasticity in chronic aphasia. BMC Biol 2:20.

Moore BC, Huss M, Vickers DA, Glasberg BR, Alcantara JI (2000) A test for the diagnosis of dead regions in the cochlea. Br J Audiol 4:205-224.

Norena AJ, Eggermont JJ (2003) Changes in spontaneous neural activity immediately after an acoustic trauma: implications for neural correlates of tinnitus. Hear Res 183:137-153.

Norena AJ, Micheyl C, Chery-Croze S, Collet L (2002) Psychoacoustical characterization of the tinnitus spectrum: implications for the underlying mechanism of tinnitus. Audiol Neurootol 7:358-369.

Rajan R, Irvine DR (1998) Neuronal responses across cortical field A1 in plasticity induced by peripheral auditory organ damage. Audiol Neurootol 3:123-144.

Sarnthein J, Stern J, Aufenberg C, Rousson V, Jeanmonod D (2006) Increased EEG power and slowed dominant frequency in patients with neurogenic pain. Brain 129:55-64.

Scherg M, Ille N, Bornfleth H, Berg P (2002) Advanced tools for digital EEG review: virtual source montages, whole-head mapping, correlation, and phase analysis. J Clin Neurophysiol 19:91-112.

Singer W (1999) Neuronal synchrony: a versatile code for the definition of relations? Neuron 24:49-65, 111-125.

Steriade M (2006) Grouping of brain rhythms in corticothalamic systems. Neuroscience 137:1087-1106.

Weisz N, Moratti S, Meinzer M, Dohrmann K, Elbert T (2005) Tinnitus perception and distress is related to abnormal spontaneous brain activity as measured by magnetoencephalography. PLoS Med 2:e153.

Weisz N, Hartmann T, Dohrmann K, Schlee W, Norena A (2006) Highfrequency tinnitus without hearing loss does not mean absence of deafferentation. Hear Res 222:108-114. 